\title{
Poster: Sensing on Ubiquitous Surfaces via Vibration Signals
}

\author{
Jian Liu ${ }^{\dagger}$, Yingying Chen ${ }^{\dagger}$, Marco Gruteser ${ }^{\S}$ \\ †Stevens Institute of Technology, Hoboken, NJ 07030, USA \\ ${ }^{\S}$ Rutgers University, North Brunswick, NJ 08902, USA \\ $\dagger\{$ jliu28, yingying.chen\}@stevens.edu, `gruteser@winlab.rutgers.edu
}

\begin{abstract}
This work explores vibration-based sensing to determine the location of a touch on extended surface areas as well as identify the object touching the surface leveraging a single sensor. It supports a broad array of applications through either passive or active sensing using only a single sensor. In the passive sensing, the received vibration signals are determined by the location of the touch impact. This allows location discrimination of touches precise enough to enable emerging applications such as virtual keyboards on ubiquitous surfaces for mobile devices. Moreover, in the active mode, the received vibration signals carry richer information of the touching object's characteristics (e.g., weight, size, location and material). This further enables our work to match the signals to the trained profiles and allows it to differentiate personal objects in contact with any surface. We evaluated extensively in the use cases of localizing touches (i.e., virtual keyboards), object localization and identification. Our experimental results demonstrate that the proposed vibration-based solution can achieve high accuracy, over 95\%, in all these use cases.
\end{abstract}

\section{INTRODUCTION}

As the form factor of our mobile and wearable devices shrinks, there exists an increasing need to support interaction beyond the confines of the device itself. One approach to address this challenge is to support convenient interaction through sensing approaches that capture input from other surfaces, without directly touching the device. Such input usually comes in the form of touches, but we consider a broad interpretation that goes beyond a human touch and includes objects touching these surfaces.

Recently, several research teams have developed gesture and activity recognition techniques that rely solely on measurable changes of the radio-frequency environment. However, these systems are affected by other changes in the surroundings that affect signal propagation. Another direction for extending interactions is using acoustic signals. They have been used to track phone movements, and recognize keystrokes on a nearby paper keyboard (e.g., [1]). The accuracy of acoustic user interaction declines sharply in noisy environments. More related are two recent studies: Toffee [2] us-

Permission to make digital or hard copies of part or all of this work for personal or classroom use is granted without fee provided that copies are not made or distributed for profit or commercial advantage and that copies bear this notice and the full citation on the first page. Copyrights for third-party components of this work must be honored. For all other uses, contact the owner/author(s).

MobiCom'16 October 03-07, 2016, New York City, NY, USA

(C) 2016 Copyright held by the owner/author(s).

ACM ISBN 978-1-4503-4226-1/16/10.

DOI: http://dx.doi.org/10.1145/2973750.2985260

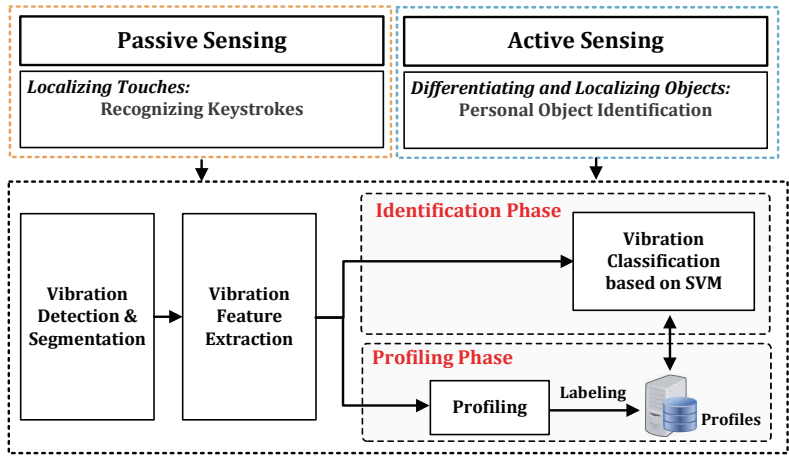

Figure 1: The architecture of our system.

es acoustic time-of-arrival correlation to determine the direction of touches on a surface with respect to a device relying on multiple piezoelectric sensors. Touch \& Activate [3] actively generates acoustic signals and records the sound patterns to identify how a user touches a small object with the vibration speaker and piezo-electric microphone directly attached to the object. These early studies are limited to devices with four well-separated sensors or support limited sensing distances.

In the quest for a touch sensing technique that is robust to environmental noise and can operate on surfaces constructed from a broad range of materials, we explore a different approach by sensing physical vibrations. The impact of a touch on a surface such as a table or door causes a shockwave to be transmitted through the material that can be passively detected with accelerometers or more sensitive piezo vibration sensors. Moreover, when a vibrator $a c$ tively excites a surface resulting in the alteration of the shockwave propagation, the presence of the object in contact with the surface can thus be sensed. This work supports generalized vibration sensing based on a low cost single sensor prototype that can receive vibration signals in both passive and active sensing scenarios. It can be attached to any solid surfaces such as a table or a door and sense touching objects. The main contributions of this work are summarized as follows:

- Exploring vibration-based sensing as a powerful touch and object sensing alternative that does not require conductive materials and is robust to acoustic noise.

- Extending passive vibration sensing to allow distinguish touches on any surface using a single receiver that can precisely work for an imaginary/paper keyboard.

- Exploring the capabilities of active vibration-based sensing in applications such as differentiating objects placed on a surface, as well as locating these objects. 


\section{SYSTEM DESIGN}

\subsection{System Overview}

As illustrated in Figure 1, the vibration-based sensing could be separated into Passive Sensing and Active Sensing depending on whether the vibration source is known to the system. The proposed work can support both types of sensing and facilitate different touch-based applications. It takes as input time-series amplitude measurements of vibration signals from a vibration receiver. After receiving the vibration signals, the system performs Vibration Detection \& Segmentation to detect and obtain the useful segment of the received vibration signals. Next, the system utilizes the Vibration Feature Extraction to extract the unique vibration feature (e.g., power spectrum density) from the segmented signals in the frequency domain. Next, the extracted vibration features are used by $\mathrm{t}$ wo phases: profiling and identification. In the profiling phase, the extracted features are considered to be the unique signature corresponding to the characteristics of the object's touches on the medium. These features are labeled with corresponding ground truth and saved to build an object profile. In the identification phase, the collected vibration samples are used to extract vibration features, which serve as inputs to a vibration classifier via Vibration Classification based on SVM to identify the target object and determine its location.

\subsection{Vibration Source}

The vibration signals collected in the passive sensing are generated by unknown vibration sources depending on specific application needs (e.g., tapping on the surface). In active sensing, the proposed system utilizes a vibrator to generate vibration. Specifically, the frequencies of the vibration signals we used increase logarithmically with time. The time duration of the generated vibration signal is set to $1 s$. Additionally, we empirically choose a relatively low frequency range (i.e., from $300 \mathrm{~Hz}$ to $12 \mathrm{kHz}$ ) to support a larger sensing area.

\subsection{Vibration Detection \& Segmentation}

After receiving vibration signals, the proposed work utilizes an energy-based approach to detect and determine the segment of useful vibration signals. In particular, it calculates the short time energy levels of the received vibration signals by accumulating the square of their amplitudes in a sliding time window. We then use a threshold-based approach to segment the useful vibration signals. The segmented vibration signal is then normalized with respect to the maximum amplitude of each.

\subsection{Vibration Feature Extraction}

Each transmission medium can be considered as a frequency selective channel for vibration signals resulting in different power and amplitude for the received vibration signal in the frequency domain. We thus choose vibration features based on the power of received vibration signals in the frequency domain. In particular, we utilize the power spectral density (PSD) of the received vibration signals as the basis for feature extraction. It could reflect the power distribution of the sensed vibration signals at each specific frequency, which can well capture the attenuation and interference effects influenced by vibration source, propagation medium, and objects contacting the medium surface.

\subsection{Vibration Classification}

During the profiling phase, the proposed work constructs a set of object profiles with the vibration features (i.e., PSD) by labeling vibration signals collected from various touch-based applications.

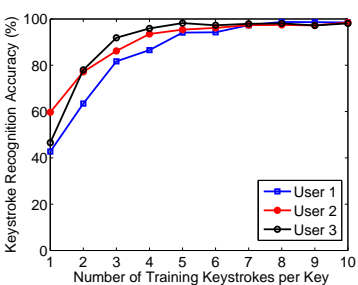

(a)

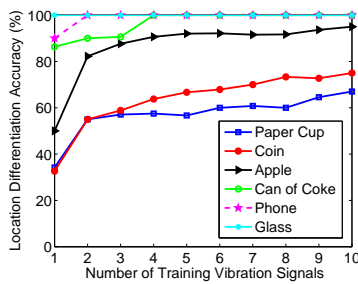

(b)
Figure 2: Performance evaluation: (a) keystroke recognition accuracy for different users with different sizes of training set and (b) localization of 6 different objects.

In the later identification phase, when there is a vibration signal detected and segmented, We need to extract the vibration feature from the segmented signal and classify the feature by matching it to the existing object profiles. Specifically, a vibration classifier is built based on the Support Vector Machine (SVM) using LIBSVM $^{1}$ and the linear kernel function.

\section{PRELIMINARY EVALUATION}

We evaluate the performance of localizing touches (passive sensing) by identifying finger clicks/keystrokes from three participants on a virtual keyboard (illustrated by a piece of paper on the surface of a wooden desk). There are 26 alphabetic keys on three rows in the virtual keyboard. Participants are asked to randomly type on the virtual keyboard with a natural speed. Each participant types and collects vibration signals 20 times for each key. Figure 2(a) shows the overall accuracy for the keystroke recognition of three participants under different training set size. We observe that the average accuracy over three users is around $87 \%$ and $97 \%$ with 3 and 5 training keystrokes per key, respectively.

For the active sensing, we conduct experiments by placing personal objects at nine locations (i.e., $3 \times 3$ grid) on a middle-size wooden table. In the experiments, six personal objects (including a small empty paper cup of $8 \mathrm{fl} \mathrm{oz}$ capacity, an U.S quarter coin, a small apple, an iPhone 5s, an empty glass cup, and a can of coke) are chosen to represent different material, weight, and size. The distance between any two adjacent predefined locations is $5 \mathrm{~cm}$. Figure 2(b) shows the localization accuracy of six different objects under different number of training vibration signals when placed at 9 positions. We observe that heavier objects obtain better localization accuracy, and the localization accuracy increases with the growing number of training signals.

\section{ACKNOWLEDGEMENT}

This work was partially supported by the National Science Foundation Grants CNS1409767, CNS1514436 and SES1450091.

\section{REFERENCES}

[1] Junjue Wang, Kaichen Zhao, Xinyu Zhang, and Chunyi Peng. Ubiquitous keyboard for small mobile devices: harnessing multipath fading for fine-grained keystroke localization. In ACM MobiSys, pages 14-27, 2014.

[2] Robert Xiao, Greg Lew, James Marsanico, Divya Hariharan, Scott Hudson, and Chris Harrison. Toffee: enabling ad hoc, around-device interaction with acoustic time-of-arrival correlation. In ACM MobileHCI, pages 67-76, 2014.

[3] Makoto Ono, Buntarou Shizuki, and Jiro Tanaka. Touch \& activate: adding interactivity to existing objects using active acoustic sensing. In ACM UIST, pages 31-40, 2013.

${ }^{1}$ https://www.csie.ntu.edu.tw/ cjlin/libsvm/ 